
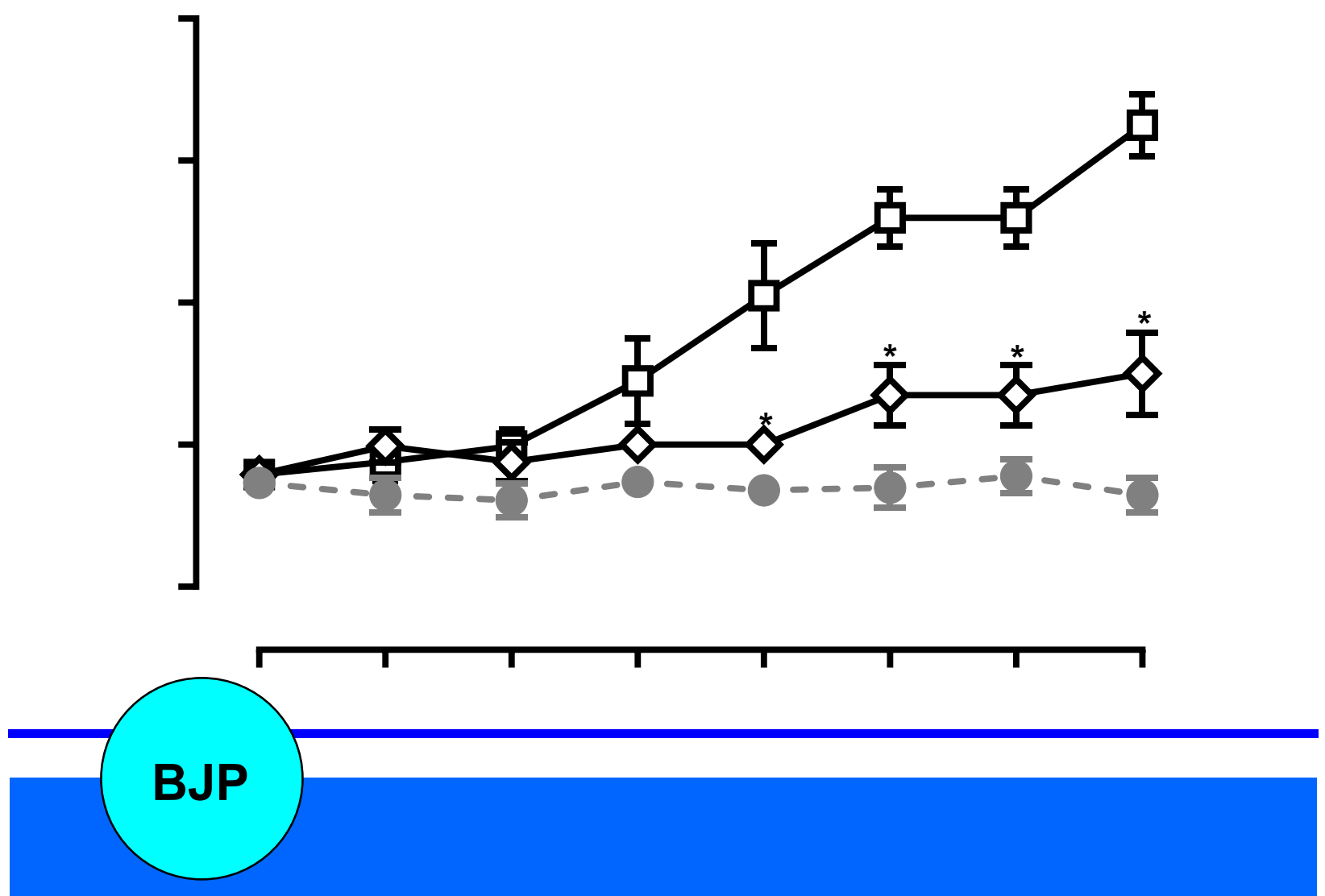

Bangladesh Journal of Pharmacology

Research Article

\title{
Effects of fisetin on oxaliplatin- induced neuropathic pain in mice
}




\title{
Effects of fisetin on oxaliplatin-induced neuropathic pain in mice
}

\author{
Hong Liu,,2, Chao-hua Wang3, Hua Yang4 and Fengjie Wang1 \\ ${ }^{1}$ School of Medicine, Hubei University for Nationalities, Enshi, Hubei, 445000, China; ${ }^{2}$ Institute of Neuropsychiatric \\ Disorder, Hubei University for Nationalities, Enshi, Hubei, 445000, China; ${ }^{3}$ Department of Neurosurgery, West \\ China Hospital, Sichuan University, Chengdu, China; ${ }^{4}$ Departments of Neurology and Pneumology, University \\ Hospital of Hubei University for Nationalities, Enshi, Hubei 445000, China.
}

\begin{tabular}{|c|c|}
\hline Article Info & \\
\hline Received: & 12 December 2014 \\
\hline Accepted: & 22 January 2015 \\
\hline Available Online: & 21 February 2015 \\
\hline DOI: $10.3329 /$ bjp. & 10i1.21203 \\
\hline $\begin{array}{l}\text { Cite this article: } \\
\text { Liu } \mathrm{H} \text {, Wang } \mathrm{CH} \\
\text { Effects of fiset } \\
\text { induced neuropa } \\
\text { Bangladesh J Pha } \\
-42 \text {. }\end{array}$ & $\begin{array}{l}\text { Yang H, Wang F. } \\
\text { on oxaliplatin- } \\
\text { hic pain in mice. } \\
\text { nacol. 2015; 10: } 138\end{array}$ \\
\hline
\end{tabular}

\section{Abstract}

Common chemotherapeutic agents such as oxaliplatin often cause neuropathic pain during cancer treatment in patients. Such neuropathic pain is difficult to treat and responds poorly to common analgesics, which represents a challenging clinical issue. Fisetin is a naturally occurring flavonoid and this study tested the potential anti-hyperalgesic effects of fisetin in a mice model of oxaliplatin-induced neuropathic pain. Fisetin (1-4 $\mathrm{mg} / \mathrm{kg}$, i.p.) did not significantly alter the mechanical hypersensitivity in oxaliplatin-treated mice but produced a dose-dependent anti-hyperalgesic effect during repeated treat-ment. Repeated treatment with fisetin also prevented chronic neuropathic pain-induced depressive-like behavior in a forced swimming test. Both the antihy-peralgesic and the antidepressant-like effects of fisetin can be blocked by a selective 5-HT1A receptor antagonist WAY100635 $(1 \mathrm{mg} / \mathrm{kg})$. Together, these results demonstrate that fisetin has significant analgesic efficacy against chronic neuropathic pain, which could be a useful analgesic in the manage-ment of neuropathic pain.

\section{Introduction}

Chemotherapy as a standard treatment for various cancers often leads to a form of unique peripheral neuropathy, which is characterized by provoked and ongoing pain and is increasingly considered as a serious side effect associated with certain chemotherapeutic agents, including taxanes, platinum agents (e.g., oxaliplatin) and vinca alkaloids. Such a side effect varies substantially from $30-75 \%$ in cancer patients who receive chemotherapy, depending on the treatment regimens. Importantly, pain is not the only symptom, and other common peripheral sensory symptoms include paresthesias and dysesthesias, numbness and tingling, and sensitivity to touch and temperature. Sometimes motor symptoms are also reported, including weakness and gait and balance disturbances (Visovsky et al., 2007). Unfortunately, this kind of neuropathic pain is only partially reversible even long after the cessation of treatment and in some rare cases damage can be permanent. Currently, no effective pharmacotherapy is available that can be considered safe and widely useful for the clinical control of chemotherapy-induced neuropathic pain. Therefore, the development of alternative effective analgesics is in dire need in the clinic.

Fisetin (3, 3', 4', 7-tetrahydroxyflavone) is a flavonoid rich in strawberries and other edible fruits or vegetables (Ross et al., 2002). It has a wide variety of pharmacological activities such as antioxidant (Khan et al., 2013), anti-inflammatory (Tordera et al., 1994), anti-allergic (Cheong et al., 1998), cancer chemopreventive (Ravichandran et al., 2011), neuroprotective activities (Patel et al., 2012) and antidepressant-like activities (Zhen et al., 2012). Because antidepressants are frequently used to treat persistent and neuropathic pain, this study examined the potential antinociceptive actions of fisetin 
in a mice model of chronic neuropathic pain. As monoaminergic system including serotoninergic system is critically involved in pain modulation and processing, the potential pharmacological mechanism underlying the antinociceptive effects of fisetin was also studied.

\section{Materials and Methods}

\section{Animals}

Male C57BL/6 mice weighing 16-22 g (Huazhou University of Science and Technology, Wuhan, China) were acclimated to the temperature, humidity and lighting (12 hours light/dark cycle, lights on at 7:00 AM) controlled vivarium and housed in groups of four for at least one week before testing. The animals had free access to dietary food and water except during the test sessions. All animal experimental protocols were approved by the local Institutional Animal Care and Use Committee, Hubei University of Nationalities. Animals were maintained and experi-ments were conducted in accordance with the Guide for the Care and Use of Laboratory Animals (8th edition, Institute of Laboratory Animal Resources on Life Sciences, National Research Council, National Acade-my of Sciences, Washington DC). All efforts were made to minimize animal suffering and to reduce the number of animals used.

\section{Drugs}

Oxaliplatin and fisetin (Sigma-Aldrich, St. Louis, MO, USA) were dissolved in 5\% dextrose $(1 \mathrm{mg} / \mathrm{mL})$ and prepared fresh for daily use. WAY100635 (SigmaAldrich, St. Louis, MO, USA) was dissolved in $0.9 \%$ saline. All injections were given intraperitoneally in a volume of $1 \mathrm{~mL} / 100 \mathrm{~g}$ of body weight. After habituation to the test environment and baseline measurements of pain sensitivity, mice were randomized to two treatment conditions of either oxaliplatin $(3.0 \mathrm{mg} / \mathrm{kg})$ or vehicle (5\% dextrose). Using injection volume of 10 $\mathrm{mL} / \mathrm{kg}$, mice were treated with daily administration for 5 days, followed by 5 days of rest, for two weekly cycles. Total cumulative dose of $30 \mathrm{mg} / \mathrm{kg}$ oxaliplatin over a total of ten injections was used.

\section{Mechanical hyperalgesia measurement}

Mechanical hyper-algesia was assessed prior to and 1 day after the last oxaliplatin treatment using Von Frey filaments of varying forces $(0.07-4.0 \mathrm{~g})$ applied to the mid-plantar surface of the right hind paw, with each application held until curved for $6 \mathrm{sec}$ using the updown method (Dixon, 1980). Mice were placed in individual Plexiglas compartments atop of a wire grid floor suspended $50 \mathrm{~cm}$ above the laboratory bench top and acclimated to the environment for $30 \mathrm{~min}$ prior to each test session. For the time course studies, baseline von Frey filament measurement was immediately followed by an injection of fisetin, and then the paw withdrawal threshold was measured every 10 min until the drug effect dissipated to a point that the paw withdrawal threshold was not significantly different from the pre-drug data. For studies that involved repeated treatment, fisetin was administered twice daily and pain threshold was mea-sured 4 hours after the first daily injection. In studies that test the effect of the antagonist WAY100635, drug was administered 10 min prior to daily fisetin treatment and pain measurement was conducted 4 hours after fisetin injection.

\section{Forced swimming test (FST)}

FST was conducted accor-ding to the reported procedure (Porsolt et al., 1977). Briefly, mice were individually plunged into vertical Plexiglas cylinders (height: $25 \mathrm{~cm}$; diameter: $10 \mathrm{~cm}$ ), which were filled with water (depth: $10 \mathrm{~cm}$; tempera-ture: $25 \pm 1^{\circ} \mathrm{C}$ ), for $6 \mathrm{~min}$. Mice were deemed immobile when they floated passively, without motion except for making only necessary movements to keep their heads above the water. The immobility time was recorded during the last $4 \mathrm{~min}$ of the total $6 \mathrm{~min}$ trial.

\section{Locomotor activity test}

Commercially available appara-tus was used to measure the locomotor activity of naïve mice treated with vehicle or fisetin (Shandong Acade-my of Medical Sciences, China), which consisted of four acrylic boxes and in each box there was one pyroelectric infrared sensor $4 \mathrm{~cm}$ above the floor. The sensor could detect the movements of the mice through infrared radiation. The apparatus recorded only gross move-ments of the mice, whereas small movements such as gnawing or grooming could not be differentiated and recorded.

\section{Data analyses}

For the antinociceptive studies, data were presented as paw withdrawal threshold (grams) plotted as a function of time (min or days), respectively. Data were analyzed by two-way repeated measures analysis of variance (ANOVA) (time $\times$ fisetin treatment or time $\times$ oxaliplatin treatment) followed by post hoc Bonfe-rroni test. For the FST data, immobility time was analyzed using oneway ANOVA followed by post hoc Bonferroni test. For the locomotion tests, data were analyzed with one-way ANOVA followed by post hoc Bonferroni test. Differences with $\mathrm{p}<0.05$ were consider-ed statistically significant.

\section{Results}

Chronic oxaliplatin treatment $(3 \mathrm{mg} / \mathrm{kg}$ ) led to marked mechanical hyperalgesia in mice as measured by von 


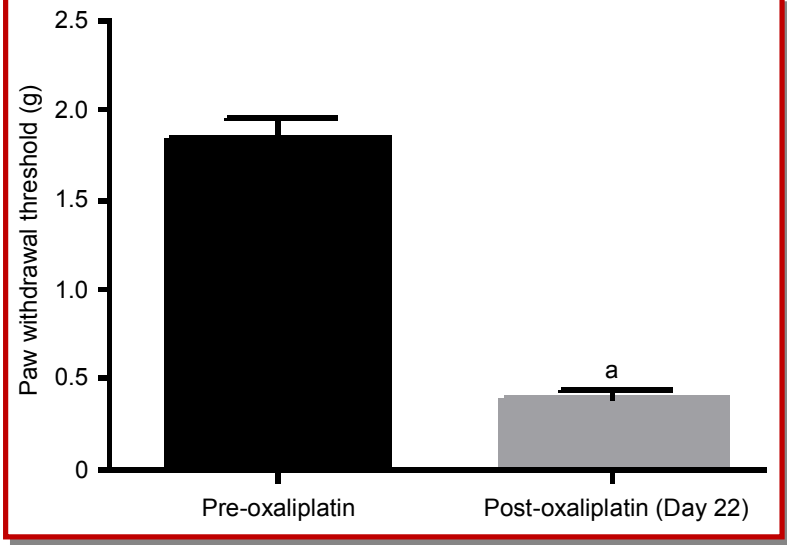

Figure 1: Paw withdrawal thresholds before and after oxaliplatin treatment in mice ( $\mathrm{n}=8$ per group). ${ }^{\text {a }} \mathrm{p}<0.001$ as compared to pre-oxaliplatin measurements

Frey filament (Figure 1). Paired t-test revealed that oxaliplatin treatment produced a significant decrease in the paw withdrawal threshold $(\mathrm{t}(7)=11.24, \mathrm{p}<0.0001)$. In addition, repeated test every $10 \mathrm{~min}$ over a period of $100 \mathrm{~min}$ did not alter the hyperalgesic condition, which remained significantly lower than the baseline measurement prior to oxaliplatin treatment (left, Figure 2). Twoway ANOVA revealed significant main effects of oxaliplatin treatment $(\mathrm{F}[3,21]=94.3, \mathrm{p}<0.0001)$ and time $(\mathrm{F}[9,63]=32.74, \mathrm{p}<0.0001)$. Acute fisetin treatment within the dose range tested $(1-4 \mathrm{mg} / \mathrm{kg})$ failed to significantly alter the paw withdrawal threshold in mice (left, Figure 2). Two-way ANOVA failed to reveal significant main effect of fisetin treatment $(F[9,126]=$ $1.78, \mathrm{p}>0.05)$. Interestingly, twice daily treatment with fisetin progressively reduced mechanical hyperalgesia (right, Figure 2). Two-way ANOVA revealed significant main effects of fisetin treatment $(\mathrm{F}[2,14]=48.48$, $\mathrm{p}<0.0001)$ and fisetin $\times$ day interaction $(\mathrm{F}[14,98]=9.18$, $\mathrm{p}<0.0001)$. Multiple comparison analysis found that the paw withdrawal threshold was significantly increased starting from day 6 at the daily dose of $2 \mathrm{mg} / \mathrm{kg}$ and starting from day 4 at the daily dose of $4 \mathrm{mg} / \mathrm{kg}$. At the dose of $4 \mathrm{mg} / \mathrm{kg}$, the paw withdrawal threshold was completely reversed 8 days after daily treatment.

In order to understand the receptor mechanism underlying the anti-hyperalgesic actions of fisetin, a dose of the selective $5-\mathrm{HT}_{1 \mathrm{~A}}$ receptor antagonist WAY100635 $(1.0 \mathrm{mg} / \mathrm{kg})$ was studied in combination with $4 \mathrm{mg} / \mathrm{kg}$ fisetin (Figure 3). Daily treatment with WAY100635 significantly and almost completely prevented the effect of fisetin for progressively increasing the pain withdrawal threshold. Two-way ANOVA revealed that there were significant main effects of WAY100635 treatment $(\mathrm{F}[1,7]=39.36, \mathrm{p}<0.0001)$ and WAY100635 $\times$ day interaction $(F[7,49]=8.24, p<0.0001)$. Post hoc analysis found that the anti-hyperalgesic effect of fisetin was significantly decreased at days 5-8.

Persistent neuropathic pain induced by oxaliplatin led to significant depressive-like behavior as demonstrated by increased immobility time in the FST test (left,

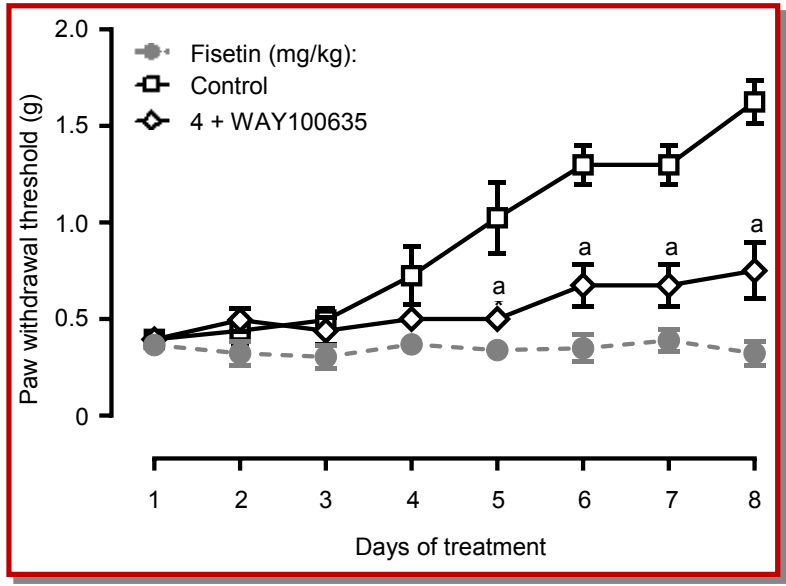

Figure 3: Effect of WAY100635 on $4 \mathrm{mg} / \mathrm{kg}$ fisetin-induced anti -hyperalgesia in mice ( $\mathrm{n}=8$ per group). a $\mathrm{p}<0.05$ as compared to corresponding $4 \mathrm{mg} / \mathrm{kg}$ fisetin data

Figure 4). Interestingly, repeated fisetin treatment not only reverted such a neuropathic pain condition, it also

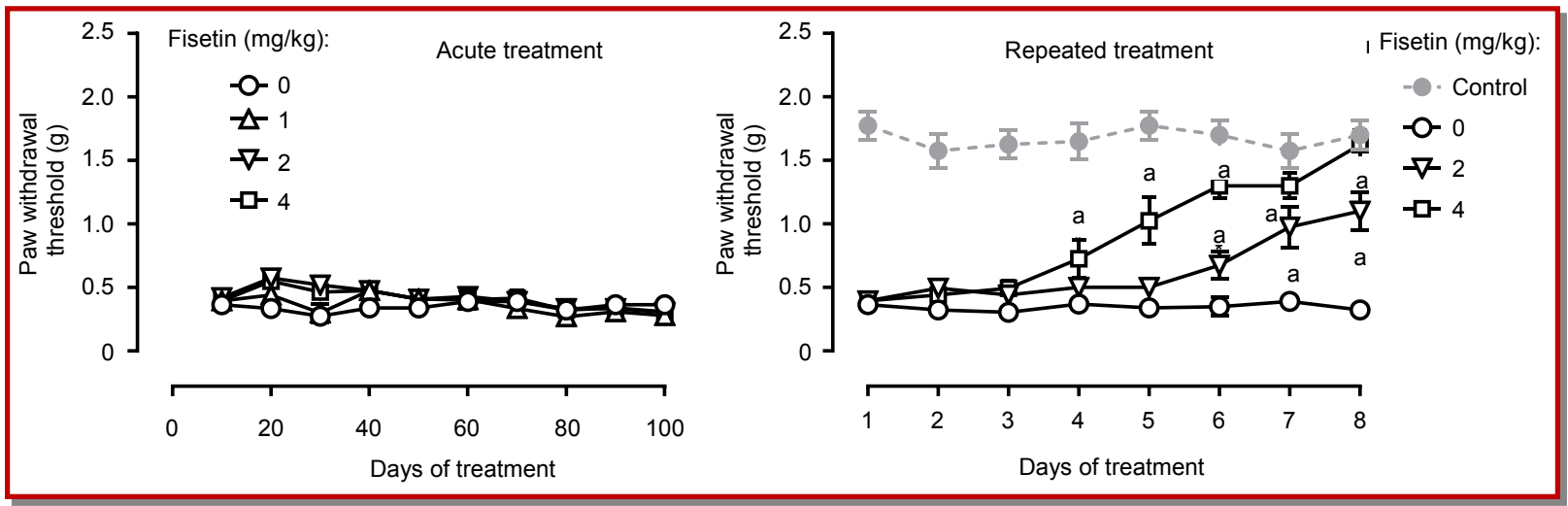

Figure 2: Effect of acute (left) and repeated (right) fisetin treatment on measure of mechanical hyperalgesia in oxaliplatin-treated mice ( $\mathrm{n}=8$ per group). a $\mathrm{p}<0.05$ as compared to corresponding control data 


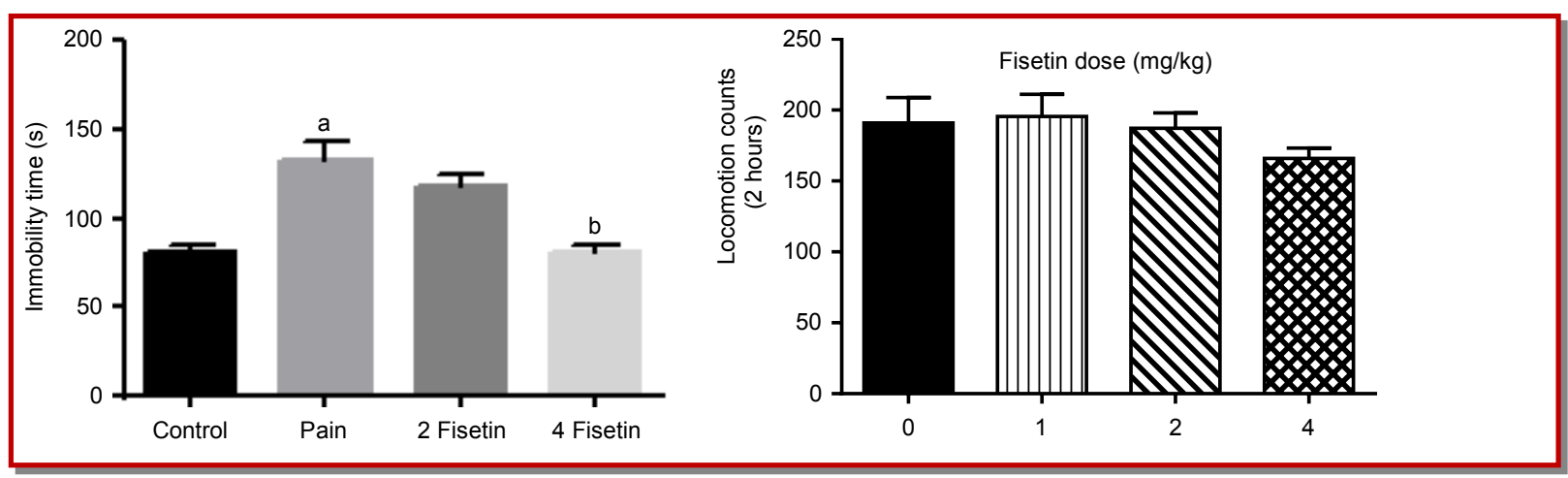

Figure 4: Effect of repeated fisetin treatment on the duration of immobility in FST in mice (left) and acute fisetin treatment on locomotor activity (right); $\mathrm{n}=8$ per group; a $<<0.0001$ as compared to non-pain control mice; ${ }^{\mathrm{p}} \mathrm{p}<0.0001$ as compared to oxaliplatintreated mice

reverted the depressive-like behavior, demonstrating antidepressant-like activity. One-way ANOVA revealed a significant effect of fisetin dose $(\mathrm{F}[3,28]=14.46$, $\mathrm{p}<0.0001)$. Post hoc analysis found that the oxaliplatintreated mice demonstrated a significantly higher immobility time as compared to non-treated control mice, and that repeated $4 \mathrm{mg} / \mathrm{kg}$ fisetin-treated mice that also received oxaliplatin treatment demonstrated a significantly lower immobility time.

The potential effect of fisetin treatment on the general locomotor activity in naïve mice was also examined (right, Figure 4). It was found that fisetin failed to significantly alter the spontaneous locomotor activity between the doses of 1 and 4. One-way ANOVA found a significant effect of fisetin dose $(\mathrm{F}[3,28]=0.9$, $\mathrm{p}>0.05)$. Post hoc analysis revealed no significant differences.

\section{Discussion}

In this study, it was found that a naturally occurring flavonoid, fisetin, produced robust anti-hyperalgesic effect in a mouse model of chemotherapy-induced neuropathic pain during repeated but not acute treatment. Along with this antinociceptive effect, repeated fisetin also prevented the development of depressive-like behavior in the mice with neuropathic pain. Then the potential pharmacological mechanism underlying the observed antinociceptive action of fisetin was examined and it was discovered that the effect was primarily mediated through serotoninergic $5-\mathrm{HT}_{1 \mathrm{~A}}$ recetors. All these data support the potential utility of using fisetin for the control of chronic pain such as chemotherapy-induced neuropathic pain.

Some cancer chemotherapeutic agents including oxaliplatin can cause peripheral and cranial neuropathy in a subpopulation of cancer patients. Several animal models of chemotherapeutic agent-induced neuropathy was developed to mimic this clinical abnormality. Rodents treated with chemotherapeutic agents typically develop thermal and mechanical hyperalgesia. In consistency with the literature, it was found that mice treated with $3 \mathrm{mg} / \mathrm{kg}$ intermittently developed a reliable mechanical hyperalgesia as measured by von Frey filament test. When administered acutely, it was found that fisetin produced no significant effect in decreasing mechanical hyperalgesia. Interestingly, twice daily treatment with fisetin produced a progressive and clear antinociceptive action in a dose-dependent manner. At larger doses it completely reversed the mechanical hyperalgesia. Importantly, this elevated mechanical pain threshold appears to be a curative effect, as the measurement was conducted 4 hours after fisetin administration. Further experiment can conclusively address this question by extended pain testing with no more fisetin treatment. It is important to note that the observed anti-hyperalgesic effects were not due to general behavioral suppression because fisetin did not significantly alter the locomotor activity in mice even at the largest tested dose. It was also observed that fisetin also prevented the development of depressive-like symptoms as evidenced by reduced immobility duration in the FST, a widely used mice model for the screening of antidepressants. Because fisetin possesses antidepressant-like activity in healthy animals (Zhen et al., 2012), this effect could be due to its antinociceptive action, its antinociceptive action or more likely the combination of both effects. This could be a highly useful feature as a novel analgesic given the high prevalence of comorbidity of chronic pain and depression (Li, 2014).

In order to begin to understand the pharmacological mechanisms of fisetin-induced antinociception, next experiment examined the involvement of $5-\mathrm{HT}_{1 \mathrm{~A}}$ receptors because $5-\mathrm{HT}_{1 \mathrm{~A}}$ receptors are critically involved in the modulation of chronic pain (Colpaert, 2006). It was found that pharmacological blockade of $5-\mathrm{HT}_{1 \mathrm{~A}}$ receptors almost completely prevented repeated fisetininduced antinociception. This finding is valuable in the guidance of future studies examining other effects fisetin. Despite these results, other mechanisms may 
also participate in fisetin-induced pain modulation, particular its antioxidant and anti-inflammatory activeties (Tordera et al., 1994; Khan et al., 2013).

In summary, this study for the first time demonstrated that fisetin has potent antinociceptive and antidepressant-like effects in a mouse model of chemotherapeutic agent-induced neuropathic pain, without apparent adverse effects (motor impairment).

\section{Acknowledgement}

This work was supported by the Key Subject of Hubei Province (Forestry).

\section{References}

Cheong H, Ryu SY, Oak MH, Cheon SH, Yoo GS, Kim KM. Studies of structure activity relationship of flavonoids for the anti-allergic actions. Arch Pharm Res. 21998; 1: 478-80.

Colpaert FC. 5-HT(1A) receptor activation: New molecular and neuroadaptive mechanisms of pain relief. Curr Opin Investig Drugs. 2006; 7: 40-47.

Dixon WJ. Efficient analysis of experimental observations. Annu Rev Pharmacol Toxicol. 1980; 20: 441-62.

Khan N, Syed DN, Ahmad N, Mukhtar H. Fisetin: A dietary anti-oxidant for health promotion. Antioxid Redox Signal.
2013; 19: 151-62.

Li JX. Pain and depression comorbidity: A preclinical perspective. Behav Brain Res. 2015; 276: 92-98.

Patel MY, Panchal HV, Ghribi O, Benzeroual KE. The neuroprotective effect of fisetin in the MPTP model of Parkinson's disease. J Parkinsons Dis. 2012; 2: 287-302.

Porsolt RD, Bertin A, Jalfre M. Behavioral despair in mice: A primary screening test for antidepressants. Arch Int Pharmacodyn Ther 1977; 229: 327-36.

Ravichandran N, Suresh G, Ramesh B, Siva GV. Fisetin, a novel flavonol attenuates benzo(a)pyrene-induced lung carcinogenesis in Swiss albino mice. Food Chem Toxicol. 2011; 49: 1141-47.

Ross JA, Kasum CM. Dietary flavonoids: Bioavailability, metabolic effects, and safety. Annu Rev Nutr. 2002; 22: 1934.

Tordera M, Ferrandiz ML, Alcaraz MJ. Influence of antiinflammatory flavonoids on degranulation and arachidonic acid release in rat neutrophils. J Biosci. 1994; 49: 235-40.

Visovsky C, Collins M, Abbott L, Aschenbrenner J, Hart C. Putting evidence into practice: Evidence-based interventions for chemotherapy-induced peripheral neuropathy. Clin J Oncol Nurs. 2007; 11: 901-13.

Zhen L, Zhu J, Zhao X, Huang W, An Y, Li S, Du X, Lin M, Wang $Q, X u Y$, Pan J. The antidepressant-like effect of fisetin involves the serotonergic and noradrenergic system. Behav Brain Res. 2012; 228: 359-66. 


\section{Your feedback about this paper}

1. Number of times you have read this paper 0

2. Quality of paper Click

3. Your comments

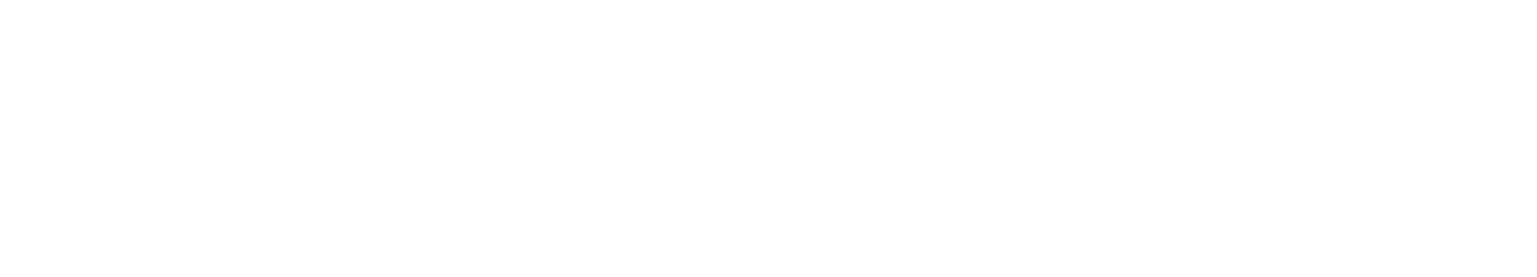

